\title{
Release Mode Dynamically Regulates the RRP Refilling Mechanism at Individual Hippocampal Synapses
}

\author{
Yujin Kim, ${ }^{-}$Unghwi Lee, ${ }^{\circledR C h u n g h o n ~ C h o i, ~ a n d ~ S u n g h o e ~ C h a n g ~}$ \\ Department of Physiology and Biomedical Sciences, Seoul National University College of Medicine, Seoul, South Korea 03080, and Neuroscience \\ Research Institute, Medical Research Center, Seoul National University College of Medicine, Seoul, South Korea, 03080
}

Synaptic strength and reliability are determined by the number of vesicles released per action potential and the availability of release-competent vesicles in the readily releasable pool (RRP). Compared with release of a single vesicle (univesicular release), multivesicular release (MVR) would speed up RRP depletion, yet whether the RRP is refilled differently during the two different release modes has not been investigated. Here, we address this question by quantitative optical imaging with an axon-targeting glutamate sensor, iGluSnFRpre. We found that hippocampal synapses preferentially release multiple vesicles per action potential at high extracellular calcium or by paired-pulse stimulation. When MVR prevails, the RRP is recovered very rapidly with a time constant of $430 \mathrm{~ms}$. This rapid recovery is mediated by dynamin-dependent endocytosis followed by direct reuse of retrieved vesicles. Furthermore, our simulation proved that the portion of retrieved vesicles that directly refill the RRP increases dramatically $(>70 \%)$ in MVR compared with that in univesicular release $(<10 \%)$. These results suggest that the contribution of rapid and direct recruitment of retrieved vesicle to the RRP changes dynamically with release mode at the level of individual synapses, which suggests a form of presynaptic homeostatic plasticity for reliable synaptic transmission during various synaptic activity.

Key words: hippocampal synapse homeostatic mechanism; multivesicular release; RRP replenishment; synaptic vesicle reuse

Significance Statement

The number of vesicles released in response to an action potential and the number of release competent vesicles in the readily releasable pool (RRP) are the fundamental determinants of synaptic efficacy. Despite its functional advantages, releasing multiple vesicles, especially at small synapses, can deplete the RRP after a couple of action potentials. To prevent failure of synaptic transmission, the RRP should be refilled rapidly, yet whether the RRP replenishment process is regulated by the release mode has not been investigated. Here, using quantitative optical glutamate imaging and simulation, we demonstrate that the contribution of the fast refilling mechanism changes with release mode at the level of individual synapses, suggesting a rapid form of presynaptic homeostatic plasticity during various synaptic activity.

\section{Introduction}

The readily releasable pool (RRP) is functionally defined as the subset of synaptic vesicles available for immediate release on stimulation. Once the RRP is depleted, presynaptic terminals can release neurotransmitters as fast as the

\footnotetext{
Received Dec. 23, 2019; revised Sep. 8, 2020; accepted Sep. 15, 2020

Author contributions: Y.K., U.L., and S.C. designed research; Y.K., U.L., and C.C. performed research; Y.K., U.L., and C.C. analyzed data; Y.K., U.L., and S.C. wrote the first draft of the paper; Y.K., U.L., and S.C. edited the paper; Y.K. and S.C. wrote the paper.

The authors declare no competing financial interests.

This work was supported by National Research Foundation of Korea Grant 2019R1A2C2089182 to S.C., Basic Science Research Program Grant 2018R1A6A3A01013515 to Y.K., and Education and Research Encouragement Fund of Seoul National University Hospital. We thank Seung Hyun Ryu for help with DNA preparation, maintenance, and transfection of cultured hippocampal neurons.

Correspondence should be addressed to Sunghoe Chang at sunghoe@snu.ac.kr.

https://doi.org/10.1523/JNEUROSCI.3029-19.2020

Copyright $\odot 2020$ the authors
}

RRP is refilled. Therefore, the number of vesicles released in response to an action potential (AP) and the rate of RRP refilling are key determinants of synaptic strength and reliability.

Conventionally, vesicles in the reserve pool (RP) are considered the major source of neurotransmitters for RRP refilling. Recent studies, however, suggest that a substantial fraction of the RRP is recycled and reused rapidly via a kissand-run mechanism, bypassing the RP-mediated pathway during single or robust repetitive AP firing at central synapses (Pyle et al., 2000; Stevens and Williams, 2000; Sara et al., 2002; Harata et al., 2006; Zhang et al., 2009). Considering the limited number of vesicles in the RRP and the slow speed of vesicle mobilization from the RP to the RRP ( $\tau=\sim$ tens of seconds) (Granseth and Lagnado, 2008), rapid reuse of vesicles could be advantageous for sustaining exocytotic activity. Despite extensive research, the prevalence of direct 
reuse and its role in rapid RRP refilling remain controversial (Fernández-Alfonso and Ryan, 2004; Granseth et al., 2007; X. S. Wu and Wu, 2009; Hua et al., 2013).

Unlike large synapses where simultaneous MVR occurs (Wadiche and Jahr, 2001; Chamberland et al., 2014), small central synapses have been generally thought to release only one vesicle in response to a single AP (univesicular release) (Bolshakov and Siegelbaum, 1995; Stevens and Wang, 1995; Hanse and Gustafsson, 2001; Silver et al., 2003; Chen et al., 2004; Biro, 2005). This classical view, however, has been challenged by other findings, including recent imaging with precise manipulation, which suggests that hippocampal synapses can release multiple vesicles when the extracellular calcium level is high or when multiple stimuli induce facilitation (Tong and Jahr, 1994; Oertner et al., 2002; Conti and Lisman, 2003; Sakamoto et al., 2018). These results imply that MVR may be a widespread mechanism that not only enhances synaptic strength and reliability but also increases the dynamic range of synaptic transmission.

Paradoxically, small synapses with $\sim 5$ vesicles/boutons in the RRP (Schikorski and Stevens, 1997; Sakamoto et al., 2018) may have to confront the danger that the releasable pool could be depleted by only a few APs under an MVR regime. Thus, a fast RRP reloading system would be crucial for the sustainability of synaptic transmission during MVR at such synapses. Direct presynaptic capacitance measurements at large synapses, such as the calyx of Held and cerebellar and hippocampal mossy fiber terminals, have shown that presynaptic activity can affect the kinetics of endocytosis and subsequent RRP replenishment (Wang and Kaczmarek, 1998; Sun et al., 2002; Ertunc et al., 2007; X. S. Wu and $\mathrm{Wu}, 2009$; Delvendahl et al., 2016). However, the reported results are contradictory, and the presynaptic conditions of these synapses may differ from those of small synapses. Recordings in hippocampal CA1 neurons showed that an increase in stimulation frequency resulted in earlier onset of vesicle reuse (Ertunc et al., 2007), but the postsynaptic interpretation of the responses during AP trains did not provide information regarding the release mode and the significance of vesicle reuse for RRP replenishment.

In this study, we investigated whether the vesicle release mode affects the mechanism of RRP replenishment. To measure glutamate release in response to a single AP at individual presynaptic terminals, we used an axon-targeting intensity-based glutamate-sensing fluorescent reporter, iGluSnFRpre. By combining our observations with simulations, we found that directly reused vesicles make a significant contribution to RRP refilling when MVR is prevalent. Since the exocytotic mode is determined by presynaptic activities, different RRP-reloading mechanisms would provide the presynaptic plasticity needed to cope with the demand for fusion-competent vesicles for immediate release.

\section{Materials and Methods}

DNA constructs. To construct the axon-targeting glutamate sensor, iGluSnFRpre, the C-terminal tail of neurexin-1(NRX-1) (kindly provided by Jaewon Ko of Yonsei University, Seoul, Korea) was added to the C-terminal region of the original iGluSnFR (iGluSnFR-NRX Ct). Then, the platelet-derived growth factor receptor (PDGFR) transmembrane domain in the original iGluSnFR was replaced, by PCR, with the transmembrane domain and C-terminal tail of NRX-1. Two different shRNA constructs against Munc13-1 (shMunc13-1_2 and shMunc131_3), kindly provided by Kenzo Hirose at the University of Tokyo, were used to silence synaptic vesicle release (Sakamoto et al., 2018). The shRNAs were expressed for at least $10 \mathrm{~d}$. Scrambled shRNA was used as a control.
Cell culture and transfection. All of the animal experiments were performed according to the Institute of Animal Care and Use Committee guidelines of Seoul National University (Seoul, Korea). Embryonic day 18 primary rat hippocampal neurons were prepared as described previously. Briefly, hippocampi were dissected, treated with papain, and triturated through a fire-polished Pasteur pipette. Dissociated cells $(150,000$ cells/60 $\mathrm{mm}$ dish) were plated on poly-D-lysine-coated coverslips in MEM supplemented with $0.6 \%$ glucose, $1 \mathrm{~mm}$ pyruvate, $2 \mathrm{~mm}$ L-glutamine, $10 \%$ FBS, and antibiotics. For autaptic culture, poly-D-lysine was sprayed on coverslips to make an island. Three to four hours after plating, the medium was replaced with Neurobasal medium (Invitrogen) supplemented with 2\% B-27 (Invitrogen), $0.5 \mathrm{~mm}$ L-glutamine, and 4 $\mu \mathrm{M}$ Ara-C. Half of the culture medium was replaced by fresh Neurobasal medium at DIVs 4,7 , and 14 . Neurons were transfected with iGluSnFR or iGluSnFRpre by the calcium-phosphate method 5-7 d after plating and used for imaging at DIV 14-20. Synapsin ImCherry or mCherry-C1 (kindly provided by Pietro De Camilli of Yale and Roger Y. Tsien of University of California at San Diego) was cotransfected for identification.

Image acquisition. Hippocampal neurons were mounted in a perfusion/stimulation chamber (Chamlide; LCI) on the stage of an Eclipse Ti$\mathrm{U}$ fluorescence microscopy (Nikon) with a $40 \times, 1.3 \mathrm{NA}$ oil lens at $35^{\circ} \mathrm{C}$ in Tyrode's solution containing the following (in $\mathrm{mm}$ ): $136 \mathrm{NaCl}, 2.5$ $\mathrm{KCl}, 10$ glucose, $10 \mathrm{HEPES}$ buffered to $\mathrm{pH} 7.3$, and $4\left(\mathrm{CaCl}_{2}+\mathrm{MgCl}_{2}\right)$, depending on experimental conditions. Stream images were acquired at $100 \mathrm{~Hz}$ with an iXon 897 EM-CCD camera $(512 \times 512,16$ bit; Andor Technologies) at cropped sensor mode of $192 \times 192$ pixels or SONA2 BV11 sCMOS camera $(1400 \times 1400$, 16 bit; Andor Technologies $)$ driven by MetaMorph Imaging Software (Universal Imaging) with a binning of 2. Two neutral density filters (ND4 and ND8) were used at a minimal exposure time to prevent instability of iGluSnFR by repeated light exposure. Field stimulation was delivered via platinum-iridium electrodes to elicit an AP $(1 \mathrm{~ms}, 20-50 \mathrm{~V}$, bipolar) using an A310 Accupulser current stimulator (World Precision Instruments). To measure spontaneous release, 10 trials of 500 frames $(5 \mathrm{~s})$ were acquired at 1 min intervals in the presence of $1 \mu \mathrm{M}$ TTX and $1 \mu \mathrm{M}$ PDBu to prevent AP firing while increasing the frequency, not the amplitude, of spontaneous release. To measure evoked responses, 30-40 trials of 40 frames $(400 \mathrm{~ms})$ were acquired in the presence of $10 \mu \mathrm{M} C N Q X$ and $100 \mu \mathrm{M} \mathrm{D}$, L-APV with an intertrial interval of $15 \mathrm{~s}$ for one or two APs, and $1 \mathrm{~min}$ for a train of APs (see Fig. 3) and after applying sucrose (see Fig. $2 K$ ). To apply sucrose, a glass pipette filled with a hypertonic solution (500 mOsm sucrose in Tyrode's solution) was placed near the ROI and briefly puffed manually. The volume applied per trial was $\sim 1 \mu \mathrm{l}$. The iGluSnFRpre response returned to baseline before the next trial began over a total of three trials.

Immunocytochemistry. For immunostaining proteins, neurons were fixed in $4 \%$ PFA for 10 min at room temperature and permeabilized with $0.25 \%$ Triton X-100 for $10 \mathrm{~min}$ at room temperature. After the permeabilization step, neurons were blocked with 3\% BSA in PBS for $30 \mathrm{~min}$ at room temperature. Then, neurons were incubated with primary antibodies in $3 \% \mathrm{BSA}$ overnight at $4^{\circ} \mathrm{C}$ and with goat anti-rabbit AlexaFluor-488-conjugated secondary antibodies (Thermo Fisher Scientific, A-11070) in 3\% BSA for $45 \mathrm{~min}$ at $37^{\circ} \mathrm{C}$. For staining iGluSnFR, anti-GFP antibodies (Abcam, ab290) were used. Anti-Dynamin1,2,3 antibodies (Synaptic Systems, 115002) were used for staining dynamin proteins. After immunostaining, cells on coverslips were mounted on a slide glass, and fluorescent images were acquired. Fluorescent intensity of dynamin1,2,3 was analyzed by using ImageJ software (National Institutes of Health).

Measurement of the recovery kinetics of the RRP. After the full size of the response to a single AP had been measured as described above for 20 trials, the neurons were stimulated by $2 \mathrm{APs}$ at $20 \mathrm{~Hz}$ at 4 [Ca] followed after a variable delay $(50,300,500,1000 \mathrm{~ms})$ by a third AP. To calculate the recovery kinetics, 20 trials with a given delay were repeated for all four delay conditions. For the experiments with Dynole and $\mathrm{NH}_{4} \mathrm{Cl}$, the full size of the evoked response was measured as control, followed by a single trial with a given delay before applying Dynasore $(100 \mu \mathrm{M})$, Dynole $34-2\left(\begin{array}{ll}1 & \mu \mathrm{M}\end{array}\right), \mathrm{NH}_{4} \mathrm{Cl}(20 \mathrm{~mm}$ replacing $\mathrm{NaCl}, \mathrm{pH} 7.3)$, or 
Table 1. Sequences of siRNAs

\begin{tabular}{lll}
\hline siRNAs & Target species & Sequences \\
\hline $\begin{array}{c}\text { 0N-TARGETplus SMARTpool Dnm1 siRNA } \\
\text { (L-088588-02-0005, Dharmacon) }\end{array}$ & Rat & GCUGAGACCGACCGAGUCA; \\
& & CCACAGAAUAUGCCGAU; \\
ON-TARGETplus SMARTpool Dnm3 siRNA & Rat & GCGUGUACCCUGAGCGUGU; \\
(L-098874-02-0005, Dharmacon) & & AGAUUCUGGUCAUUCGAAA \\
& & GUGCUAGAUCAAUCGCAU; \\
& & GGCAACAAGUAGACACGUU; \\
& & GAACUGAUCAACACGGUGA \\
\hline
\end{tabular}

latrunculin $\mathrm{B}(1$ or $5 \mu \mathrm{M})$. Another trial with the same delay was given in the presence of the drugs. In the case of Dynasore, Dynole, and latrunculin $\mathrm{B}$, neurons were incubated for $10 \mathrm{~min}$ before the last trial. The concentration of Dynasore and Dynole was carefully chosen not to impair exocytosis. For the experiments in siRNA-Dyn1,3 transfected neurons, the extra intertrial interval was provided to ascertain the full replenishment of the RRP.

Protein knockdown by siRNAs. Cultured hippocampal neurons were transfected with siRNA mixture targeting Dynamin 1 and 3 by using Lipofectamine RNAiMAX Transfection Reagent (Thermo Fisher Scientific) followed by the manufacturer's instructions at DIV4 and DIV7. Live image acquisition, Western blot, and immunocytochemistry were performed at DIV14. The sequences of siRNAs used in this research are as in Table 1.

Electrophysiology. Whole-cell patch-clamp recordings were conducted using cultured hippocampal neurons at DIV14-DIV16. To record mEPSCs, neurons were voltage-clamped at $-70 \mathrm{mV}$ in the presence of 1 $\mu \mathrm{M}$ TTX and $10 \mu \mathrm{M}$ bicuculline by using EPC- 10 amplifier ( $3 \mathrm{kHz}$ filtering and $10 \mathrm{kHz}$ sampling) and PATCHMASTER software (HEKA Elektronik) at room temperature. Patch pipettes were pulled from borosilicate glass capillaries (Hilgenberg) to the resistance of 3-5 $\mathrm{m} \Omega$ and filled with pipette internal solution $(130 \mathrm{~mm}$ potassium gluconate, 20 mм KCl, 10 mм K-HEPES, 0.2 mм EGTA, 0.3 mм Na-GTP, 4 mм MgATP, pH 7.3, 285-290 mOsm). Tyrode's solution was used as an extracellular solution. The amplitude and frequency of mEPSC were analyzed by MiniAnalysis software (Synaptosoft).

Western blot. Neurons were lysed in $1 \%$ Triton X-100 lysis buffer (20 mм Tris-HCl, pH 8, 1\% Triton X-100, 10\% glycerol, $137 \mathrm{~mm} \mathrm{NaCl}, 2 \mathrm{~mm}$ EDTA, $1 \mathrm{~mm}$ PMSF) with a protease inhibitor cocktail (Sigma Millipore). Neuron lysates were centrifuged at $14,000 \times g$ at $4^{\circ} \mathrm{C}$ for 20 min after sonication. The supernatants were used for the cell lysate samples, and protein concentration was measured using a BCA quantitative assay kit (Thermo Fisher Scientific); $2 \times$ protein sample buffer was added to the sample $(10 \mu \mathrm{g})$, followed by a boiling step $\left(100^{\circ} \mathrm{C}, 5 \mathrm{~min}\right)$, and each sample was separated by polyacrylamide gels (6\%), then transferred to PVDF membranes (Pall Life Sciences). After protein transfer, membranes were blocked with $5 \%$ skim milk for $30 \mathrm{~min}$ at room temperature and washed by TBST. Membranes were incubated with the primary antibody overnight at $4^{\circ} \mathrm{C}$. After washing three times for $5 \mathrm{~min}$ in TBST, membranes were incubated with HRP-conjugated secondary antibody for $45 \mathrm{~min}$ at room temperature. Immunoreactivity was detected with an enhanced chemiluminescence reagent (ECL; AbClon) and LAS 4000 (GE Healthcare).

Modeling glutamate depletion in the RRP. The quantitative model focused on the outcome of vesicle redistribution after each trial of 1 AP. The difference between previous models and ours is that we paid attention to the fraction of reused (empty) and RP-originated (glutamatefilled) vesicles in the RRP. Unlike previous data acquired by trains of APs where vesicle release, fast and slow recycling takes place all at once, our data are a direct read-out of the proportion of glutamate-filled vesicles in the RRP after all the processes are completed during a sufficient intertrial interval. Thus, neither the kinetic parameter of the individual processes nor the number of different vesicle pools is under consideration in our model. Instead, four input parameters were introduced to simulate the experiments in Figure $4: \mathrm{N}_{\mathrm{RP}}$, the total number of vesicles in the RP; $\mathrm{N}_{\mathrm{RRP}}$, the total number of vesicles in the RRP; $\mathrm{Pv}$, the release probability of a vesicle in the RRP; and Pe, the proportion of endocytosed vesicles directly recruited to the RRP, with the following assumptions:

1. The amount of glutamate in every vesicle is constant.

2. The release probability of each vesicle in the RRP is constant (Pv). Since only a single AP is applied, activity-dependent changes in $\mathrm{Pv}$ do not exist.

3. The redistribution of endocytosed vesicles (fast reuse) precedes the recruitment of RP vesicles to the RRP.

4. The numbers of vesicles in the RP and the RRP $\left(\mathrm{N}_{\mathrm{RP}}\right.$ and $\mathrm{N}_{\mathrm{RRP}}$, respectively) at the beginning of each trial are constant.

5. The number of released vesicles equals the number of retrieved vesicles.

6. Vesicles behave the same regardless of the presence or absence of glutamate.

7. Spontaneous vesicle rearrangement asynchronous to AP is negligible.

When an AP arrives at the presynaptic terminal at the $\mathrm{i}^{\text {th }}$ trial, the number of synaptic vesicles released (Rrel[i]) is determined by the binomial distributions of $\mathrm{N}_{\mathrm{RRP}}$ and Pv. From Rrel[i], we obtain the number of released vesicles containing glutamate (Rglu[i]) by random sampling $\mathrm{N}_{\mathrm{RRP}}$ with the number of glutamate-filled vesicles in the RRP (RRPglu [i]) for Rrel[i] times. Rglu[i] is the value that corresponds to our empirical data. After exocytosis, the same number of vesicles (Rrel[i]) will be endocytosed. The number of vesicles directly recruited to the RRP (Vrec [i]) is acquired by random sampling Rrel[i] with a probability of Pe. The remaining vesicles (Rrel[i] - Vrec[i]) will be transferred to the RRP, and this is the same as the number of vesicles recruited from the RP to the RRP. The number of vesicles recruited from the RP to the RRP containing glutamate (Eglu[i]) can be acquired by random sampling $\mathrm{N}_{\mathrm{RP}}$ with the number of glutamate-filled vesicles in the RP (RPglu[i]) for (Rrel[i] Vrec[i]) times. In the presence of $\mathrm{NH}_{4} \mathrm{Cl}$, the endocytosed vesicles are glutamate-free. Thus, at the beginning of the next trial after complete synaptic vesicle recycling, the numbers of vesicles with glutamate in the RRP and the RP are RRPglu[i+1] = RRPglu[i] - Rglu[i] + Eglu[i] and RPglu[i +1$]=$ RPglu[i] - Eglu[i], respectively.

To generate one simulated experiment, we repeated this cycle with AP for 30 trials as it is in the empirical data in Figure 4 . We set 18 values of $\mathrm{Pv}$ in the range between 0.01 and 0.9, 20 values of Pe between 0 and 1, 4 values of $\mathrm{N}_{\mathrm{RP}}$ between 20 and 140 , and 7 values of $\mathrm{N}_{\mathrm{RRP}}$ between 4 and 7. We then repeated the simulation 1000 times with each of $10,800 \mathrm{com}-$ binations of the parameters and calculated the $\chi^{2}$ value between the empirical data and each simulated result to quantify the goodness of fit.

Data analysis. Responses from individual boutons were extracted using ImageJ (National Institutes of Health). A response amplitude exceeding 3.5 SDs of the baseline was regarded as a success. Boutons with peak formed $40 \mathrm{~ms}$ after response onset were excluded. To fit the distribution of evoked responses in Figure $2 B$, the mean and variance $(v)$ of the Gaussian fit of spontaneous responses (see Fig. 1E) were first obtained. Assuming that evoked release is multiquantal, the evoked responses were then fitted by the sum of Gaussian distributions with multiple means $(1,2$, and variance $[v, 2 v]$ ) (Boyd and Martin, 1956). To compare the response amplitudes evoked at 2 and $4[\mathrm{Ca}]$, only successive responses were included, and these were then normalized by the first response at 2[Ca]. Fast-decaying iGluSnFRS72A was used to obtain precise measurements during repetitive stimulation. Boutons with $<5$ successes were excluded for reliability. To measure calcium influx at presynaptic terminals, the GCaMP6f responses to single APs at 2 and 4 [Ca] were grouped and averaged, regardless of success/failure.

Statistical analysis. Data were analyzed with Excel (Microsoft), Igor Pro (Wavemetrics), and Origin (OriginLab). Most experiments were designed to have within-subject control, such as before and after treatment to reduce bouton-to-bouton variability. Group data from averages of multiple trials or boutons are represented as mean \pm SEM, while those from single trials are represented as mean $\pm 95 \%$ CI, with the number of experiments and boutons used in each experiment. Statistical significance was evaluated using Student's $t$ test for two-group comparison. A comparison of more than two groups were performed using oneway ANOVA and Tukey's HSD test. Differences in time-dependent 
A iGluSnFR
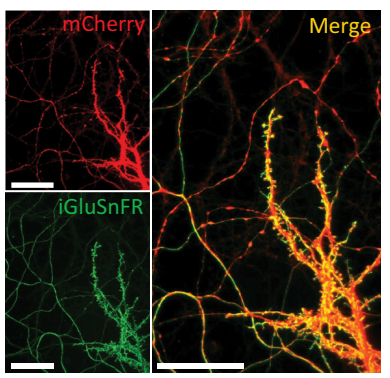

C

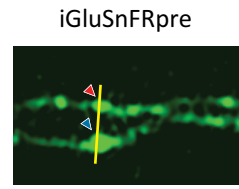

D
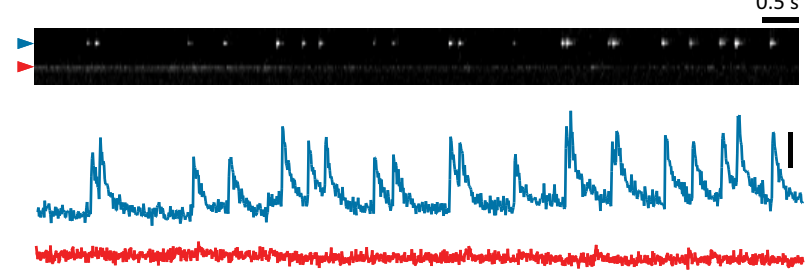

E

Spontaneous

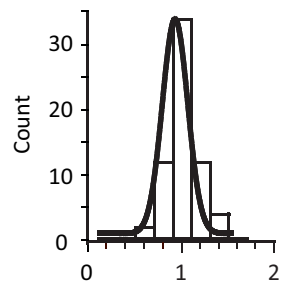

Normalized amplitude
iGluSnFRpre
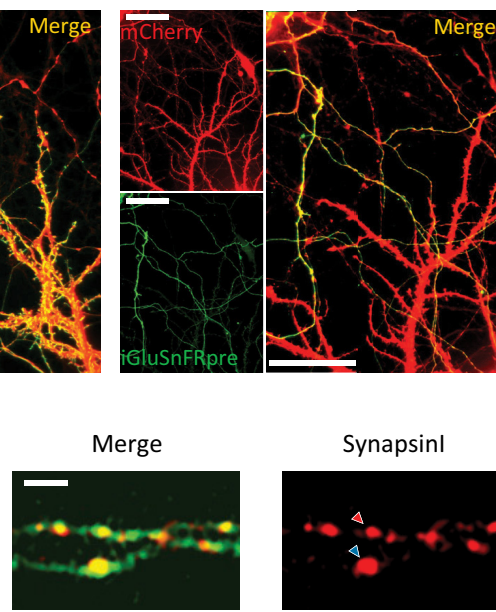

F

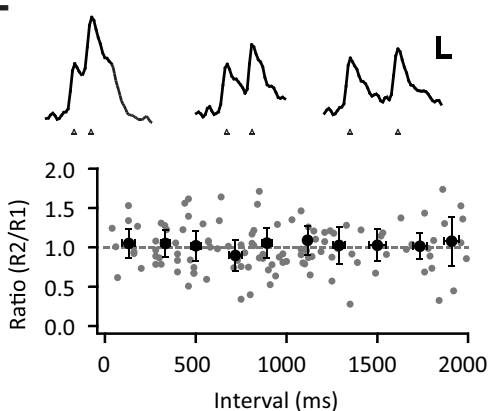

B

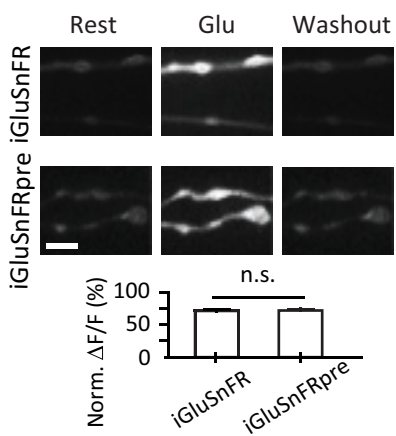

G

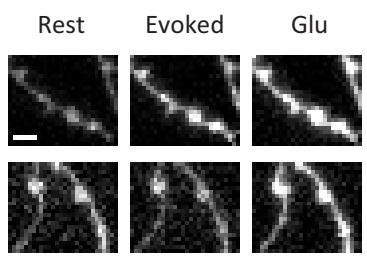

H

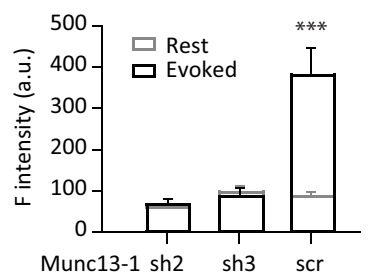

I

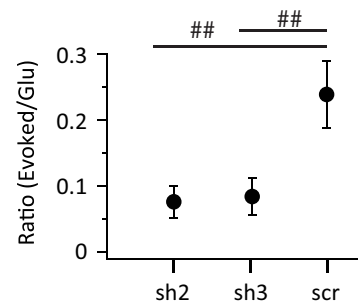

Figure 1. Imaging glutamate release at single presynaptic terminals with iGluSnFRpre. $A$, Expression patterns of iGluSnFR and iGluSnFRpre in cultured hippocampal neurons. mCherry shows the neuronal morphology. Scale bar, $40 \mu \mathrm{m}$. B, Responses of iGluSnFR and iGluSnFRpre to glutamate. Top, Representative images of boutons expressing iGluSnFR or iGluSnFRpre at rest (left), in $100 \mu \mathrm{m}$ glutamate (Glu; middle), and after washout (right). Bottom, Pooled data. $n=41$ boutons from 5 experiments (iGluSnFR), 38 boutons from 5 experiments (iGluSnFRpre). Student's $t$ test. Scale bar, $5 \mu \mathrm{m}$. C, Representative images of an axonal segment expressing iGluSnFRpre and synapsinl-mCherry. Scale bar, $5 \mu \mathrm{m}$. D, Top, Kymograph of spontaneous iGluSnFRpre responses from the axonal segment shown in C (yellow line); $1 \mu \mathrm{m}$ TTX was included to prevent spontaneous AP firing, and $1 \mu \mathrm{m}$ PDBu was included to increase the frequency of spontaneous release. Bottom, iGluSnFRpre responses from two presynaptic boutons shown in $\mathbf{C}$ (arrowheads). The response from a synaptic terminal is not detected from neighboring synapses. Scale bar, 200 a.u. $\boldsymbol{E}$, Amplitude histogram of the spontaneous response of blue trace in $\boldsymbol{D}$. Response amplitudes were normalized by the mean of the single Gaussian distribution (thick line). $\boldsymbol{F}$, Top, Examples of two spontaneous responses with various intervals. Triangles represent peak location. Scale bar: 200 a.u., $50 \mathrm{~ms}$. Bottom, Ratio of the amplitude of the second peak (R2) to the first (R1) as a function of interpeak interval. $n=120$ pairs of peaks from 21 boutons. Data are mean $\pm 95 \%$ Cl. $\mathbf{G}$, Representative images of boutons expressing either shRNA against Munc13-1 (sh2) or scrambled shRNA (scr) at rest (left), when evoked by 1 AP (middle), and in $100 \mu \mathrm{m}$ glutamate (right). Scale bar, $5 \mu \mathrm{m}$. $\boldsymbol{H}$, Evoked and rest response from boutons expressing either shRNA against Munc13-1 (sh2, sh3) or scr. $n=10$ (sh2), 12 (sh3), and 12 (scr) experiments. $p\left(\right.$ sh2) $=0.688, p(s h 3)=0.571, p(s c r)=0.000$. Data are mean \pm SEM. ${ }^{* * *} p<0.001$ (paired Student's $t$ test). $I$, The ratio of evoked response to the response in the presence of $100 \mu \mathrm{m}$ glutamate from boutons expressing different shRNA constructs. $p$ (sh2-sh3) $=0.9841 ; p($ sh2$s c r)=0.003 ; p($ sh3-scr) $=0.003 \#$ \#: $p<0.01$ (Tukey's HSD test).

changes between two conditions (see Fig. $4 F$ ) were evaluated using twoway repeated-measures ANOVA. Groups that cannot be assumed to be equally distributed (see Fig. $2 J$ ) were evaluated using the Wilcoxon signed-rank test. $p>0.05$ was regarded as not significantly different.

\section{Results}

Imaging glutamate release at single presynaptic terminals with iGluSnFRpre

We modified iGluSnFR, an intensity-based glutamate-sensing fluorescent reporter, replacing the C-terminal transmembrane domain with that of neurexin to form iGluSnFRpre. iGluSnFRpre is expressed exclusively in axonal segments and has comparable glutamate responsiveness to the parental form (Fig. 1A,B). iGluSnFRpre responses (iGlu responses) to spontaneous release were confined to individual presynaptic boutons (Fig. 1C,D). Also, the amplitude histogram was fitted by a single Gaussian distribution (Fig. 1E), implying that the unit response because of single vesicle release was reliably detected. Occasionally, we found that two spontaneous responses overlapped, and yet the sizes of the first and the second responses were not significantly different regardless of the interval between them (Fig. $1 F$ ). This suggests that iGluSnFR responds linearly to the release of at least a few vesicles without saturation, consistent with a previous study (Marvin et al., 2013). To confirm whether the iGlu response at a 

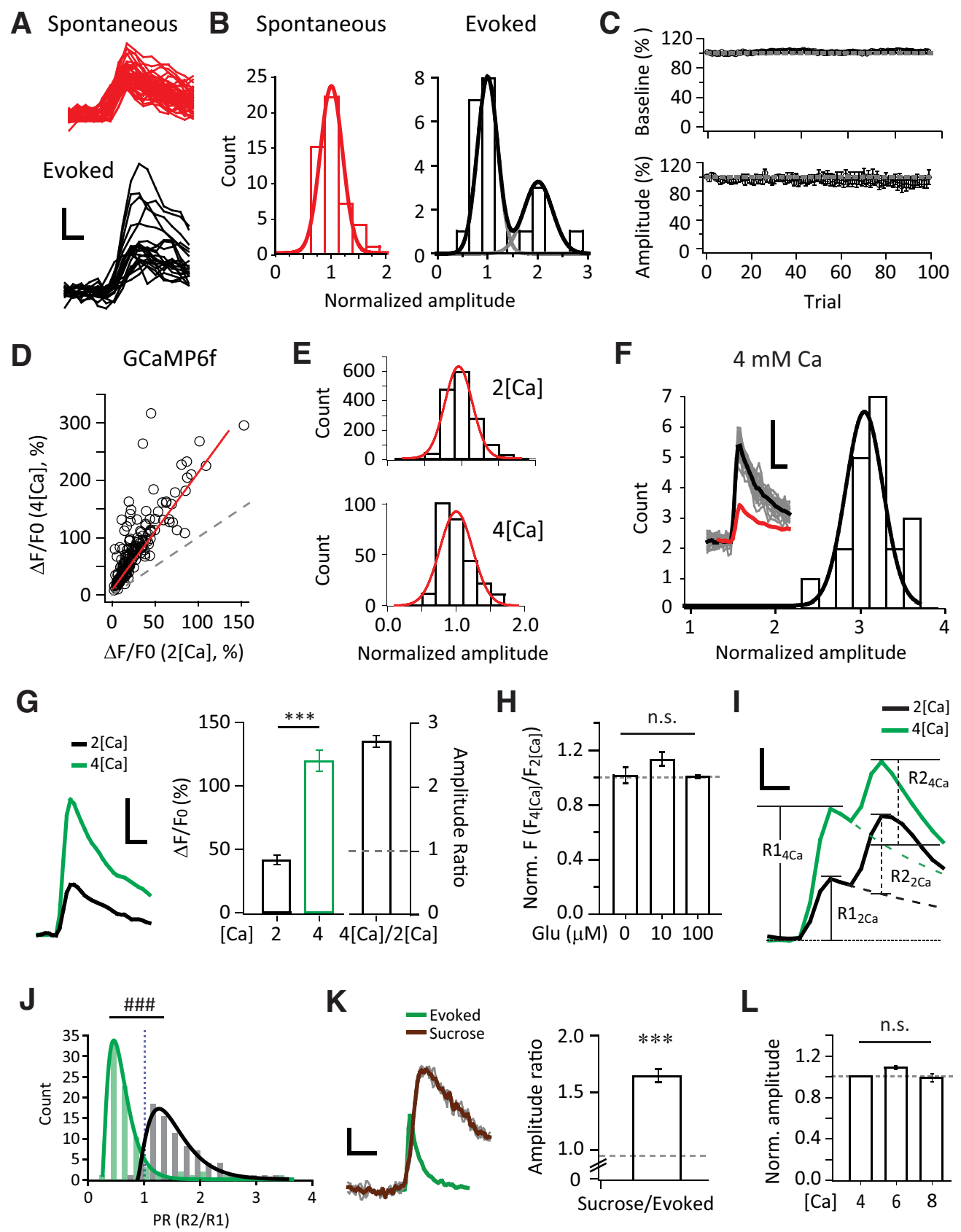

$\mathbf{L}$

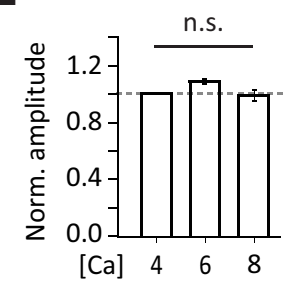

Figure 2. MVR at single synapses. $\boldsymbol{A}$, Spontaneous (red) and evoked (black) responses from a single presynaptic terminal. Calibration: $50 \% \Delta \mathrm{F} / \mathrm{F}_{0}, 20 \mathrm{~ms}$. B, Amplitude histograms of the spontaneous (red) and evoked (black) responses in $\boldsymbol{A}$. Response amplitudes were normalized by the mean of the distribution of spontaneous responses. Red trace represents a Gaussian fit to the spontaneous responses. Black trace represents the sum of two Gaussian fits for the evoked responses using the means $(1,2)$ and SDs $(\sigma, \sqrt{2} \sigma)$ derived from the fit of the spontaneous responses. C, Stability of iGlu response. Evoked responses to $1 \mathrm{AP}$ with an exposure time of $400 \mathrm{~ms}$ were imaged every $15 \mathrm{~s}$ for 100 times. Baseline (top) and response amplitude (bottom) were normalized by the average of the first five trials. D, Scatter plot of GCaMP6f responses to $1 \mathrm{AP}$ at $2[\mathrm{Ca}$ ] and $4[\mathrm{Ca}$ ]. Red line indicates linear regression (slope: $2.0 \pm 0.1$ ). Dotted line indicates $y=x . n=169$ boutons from 13 experiments. Mean \pm SEM: $28 \pm 2$ at 2[Ca], $80 \pm 4$ at 4[Ca]. E, Amplitude histogram of spontaneous responses at 2[Ca] (top) and 4[Ca] (bottom). Red traces represent the Gaussian fit of each histogram. 2[Ca]; $n=1535$ trials from 53 boutons; mean $=1 ; S D=0.20 .4[C a] ; n=276$ trials from 20 boutons; mean $=1 ; S D=0.24$. Kullback-Leibler divergence of the two fits is 0.039 . $\boldsymbol{F}$, Amplitude histogram of evoked responses at $4[\mathrm{Ca}]$. Inset, Traces of average spontaneous (red) and evoked responses (gray represents individual trials; black represents average). Calibration: $100 \% \Delta \mathrm{F} / \mathrm{F}_{0}, 50 \mathrm{~ms}$. $\mathbf{G}$, Left, Representative traces at $2[\mathrm{Ca}]$ (black) and $4[\mathrm{Ca}]$ (green) from a single bouton. Each trace represents an average of 20 responses to 1 AP. Calibration: $50 \% \Delta \mathrm{F} / \mathrm{F}_{0}, 50 \mathrm{~ms}$. Middle, Pooled graph of evoked responses; $n=103$ boutons from 9 experiments. Mean \pm SEM: $46 \pm 4$ (2[Ca]), $120 \pm 8$ (4[Ca]). $p=0.000$ by paired Student's $\boldsymbol{t}$ test. Right, Ratio of response amplitude at 4[Ca] to 2[Ca]. $\boldsymbol{H}$, iGlu response to $0,10,100 \mu \mathrm{m}$ glutamate at $4[\mathrm{Ca}$ ] normalized by that at $2[\mathrm{Ca}]$. Mean \pm SEM $=1.01 \pm 0.06$ at $0 \mu \mathrm{m} ; 1.14 \pm 0.05$ at $10 \mu \mathrm{m} ; 1.01 \pm 0.00$ at $100 \mu \mathrm{m} . n=4$ experiments. $p=0.910,0.088,0.387$, respectively (paired Student's $t$ test). $I$, Representative average responses to a pair of stimuli with $50 \mathrm{~ms}$ interval at 2[Ca] (black) and 4[Ca] (green). To calculate R2, the baseline of the response is estimated by the exponential decay of the first response (dotted line) (for details, see Materials and Methods). Calibration: $50 \% \Delta \mathrm{F} / \mathrm{F}, 25 \mathrm{~ms}$. J. Distribution of PR at different [Ca]. Solid lines indicate $\gamma$ distribution fits at 2[Ca] and 4[Ca] (shape $=2.42$, scale $=0.91)$. Median is 1.50 (2[Ca]) and 0.54 (4[Ca]). $n=78 . p=0.000 .{ }^{\# \#} p<0.001$ (paired Wilcoxon signed-rank test). $\boldsymbol{K}$, Left, Representative responses by 1 AP at 4[Ca] (evoked; green) and the application of 0.5 Osmol sucrose (sucrose; brown). Calibration: $50 \% \Delta \mathrm{F} / \mathrm{F}_{0}, 200 \mathrm{~ms}$. Right, Ratio of responses to sucrose to evoked. Mean $\pm \mathrm{SEM}=1.65 \pm 0.06 . n=40$ boutons from 4 experiments. $p=0.000$. ${ }^{* * *} p<0.001$ (paired Student's $t$ test). $L$, iGlu response at high [Ca] conditions. The data are normalized by the response at 4[Ca]. Mean $\pm 95 \%(\mathrm{Cl}=1.08 \pm 0.02$ (6[Ca]), $0.99 \pm 0.04$ (8[Ca]). $n=25$ boutons from 2 experiments. $p=0.866$ (one-way ANOVA). 
bouton reflects vesicle release from that bouton and is not contaminated by neighboring synaptic activity, we transfected neurons with an shRNA against Munc13-1, which reduced endogenous Munc13-1 by $90 \%$ (Sakamoto et al., 2018). We then triggered vesicle release in response to field stimulation (Fig. 1G-I). Compared with the control expressing scrambled shRNA, evoked responses at Munc13-1-deficient boutons were severely impaired, indicating that iGluSnFRpre is suitable for detecting exocytotic events at single presynaptic terminals.

\section{MVR at single synapses}

The univesicular release hypothesis suggests that successful synaptic vesicle release produces a one-size response regardless of stimulus strength (Bolshakov and Siegelbaum, 1995; Stevens and Wang, 1995). However, if MVR occurred, the response could increase to multiples of the unit size. To examine the exocytotic mode of hippocampal synapses, we compared spontaneous and evoked responses at the normal calcium concentration of $2 \mathrm{~mm}$ (2[Ca]; Fig. $2 A, B)$. Again, amplitude histograms of spontaneous responses fitted a Gaussian distribution. The iGlu responses evoked by single APs, however, were slightly noisy; the majority were distributed around the mean of the spontaneous responses, with a few outliers close to twice the mean (Fig. 2B). Baseline intensity and evoked response size were stable over repetitive trials (Fig. 2C), suggesting that glutamate release at $2[\mathrm{Ca}]$ is mostly univesicular with a few multivesicular events.

To further evaluate MVR, we measured iGlu responses at elevated extracellular calcium ( $4 \mathrm{~mm} ; 4[\mathrm{Ca}])$, where calcium influx in response to a single AP doubled $(2.0 \pm 0.1$ compared with 2 [Ca]; Fig. 2D). Spontaneous release was not altered (Fig. 2E; $\mathrm{SD}=0.20$ at $2[\mathrm{Ca}$ ], 0.28 at $4[\mathrm{Ca}$; Kullback-Leibler divergence: 0.039). However, most of the iGlu responses evoked at $4[\mathrm{Ca}]$ fitted a single Gaussian curve whose mean approximated a multiple of the mean of the spontaneous responses (Fig. $2 F$ ), indicating that MVR prevailed. Consistent with this, the response amplitude at $4[\mathrm{Ca}]$ was 2.6 times larger than that at $2[\mathrm{Ca}](2.6 \pm 0.10$; Fig. 2G). Since the iGlu response was independent of extracellular calcium (Fig. $2 H$ ), the increased response size is attributable to multiple vesicle release. To test whether MVR occurred in response to a physiological increase in presynaptic [Ca], we applied 2 APs at $50 \mathrm{~ms}$ intervals and compared the responses to each AP (Fig. 2I), and then calculated the potency ratio (PR), the ratio of successful second responses (R2) to successful first responses (R1). Most boutons at $2[\mathrm{Ca}](94.9 \%)$ gave a $\mathrm{PR}>1$ (mean $=1.57, \mathrm{SD}=0.54$; Fig. $2 J$ ), suggesting that the amount of glutamate released per AP was increased by neuronal activity, consistent with a previous report (Oertner et al., 2002). In contrast to the facilitation of $\mathrm{PR}$ at $2[\mathrm{Ca}]$, depression $(\mathrm{PR}<1)$ was observed at $4[\mathrm{Ca}]($ mean $=0.55, \mathrm{SD}=0.54)$. One explanation for $\mathrm{PR}$ depression is a reduction in the number of vesicles in the RRP because of the first AP. To estimate the size of the RRP, we measured the iGlu responses of the total RRP by applying a hypertonic sucrose solution (500 mOsm). The response amplitude in the hypertonic solution was 1.65 times larger than that evoked at $4[\mathrm{Ca}](1.65 \pm 0.06$; Fig. $2 \mathrm{~K})$. Raising extracellular calcium to $8[\mathrm{Ca}]$ did not significantly increase evoked responses (Fig. 2L), suggesting the existence of a limited number of release sites at presynaptic terminals. From the data collected here, we can estimate the size of the RRP as 4.3 vesicles $(2.6 \times 1.65$, assuming the evoked responses at $2[\mathrm{Ca}]$ are single vesicle events), which is consistent with previous reports (Schikorski and Stevens, 1997; Auger and Marty, 2000; Sakamoto et al., 2018). Together, these results indicate that hippocampal synapses are capable of releasing multiple vesicles in response to a single AP when the presynaptic calcium level is high.

\section{Dynamin-dependent fast recovery of the RRP}

During MVR, the RRP would be depleted after a few APs unless it was replenished rapidly. We first examined the sustainability of synaptic transmission by applying $5 \mathrm{APs}$ at $10 \mathrm{~Hz}$ (Fig. 3A,B). We used a fast decaying variant iGluSnFRpre.S72A to minimize the accumulation of successive responses (Marvin et al., 2018). iGlu responses dropped more rapidly at $4[\mathrm{Ca}]$ (where MVR prevails) than $2[\mathrm{Ca}]$ (where univesicular release prevails). The fifth response at $4[\mathrm{Ca}]$ was still comparable to the first response at 2 [Ca], suggesting that a fraction of the RRP was refilled rapidly during the AP train.

To identify the source of this fast RRP refilling and to measure its kinetics, we applied 2 consecutive $20 \mathrm{~Hz}$ APs at $4[\mathrm{Ca}]$, expecting that a substantial portion of the RRP would be released, and then delivered a third test $\mathrm{AP}$ at various times after the end of the second AP (Fig. $3 C$ ). We found that the third response increased with the length of the interval and recovered fully in $15 \mathrm{~s}$ ( $\tau_{\text {fast }}=430.9 \mathrm{~ms}, \tau_{\text {slow }}=4.11 \mathrm{~s}$; Fig. $\left.3 D, E\right)$. Strikingly, the responses recovered by up to $\sim 63.6 \%$ of the initial response within a second. To test the contribution of recycled vesicles to this recovery, we used $20 \mathrm{mM} \mathrm{NH}_{4} \mathrm{Cl}, \mathrm{pH}$ 7.3, which neutralizes the interior of synaptic vesicles and blocks reloading of glutamate into recycled vesicles. We first confirmed the potency of $\mathrm{NH}_{4} \mathrm{Cl}$ as a vesicular refilling blocker. We found that the frequency of mEPSC was dramatically reduced in the bafilomycin (Baf)-, an inhibitor of vacuolar $\mathrm{H}^{+}$-ATPase, or $\mathrm{NH}_{4} \mathrm{Cl}$ treated neurons, while mEPSC amplitude remained unchanged (Fig. $3 F$ ). We also recorded EPSCs from autaptic neurons by current injection and found that before treatment a single EPSC recorded $5 \mathrm{~s}$ after $40 \mathrm{AP}$ at $20 \mathrm{~Hz}$ was almost fully recovered (97.5\%), whereas it failed to recover in the presence of Baf and $\mathrm{NH}_{4} \mathrm{Cl}\left(31.6 \%\right.$ and $26.5 \%$ with $\mathrm{Baf}$ and $\mathrm{NH}_{4} \mathrm{Cl}$, respectively; Fig. $3 G$ ). Both results confirmed that $\mathrm{NH}_{4} \mathrm{Cl}$ is a reliable blocker of glutamate refilling. We then repeated the recovery experiment in the presence of $\mathrm{NH}_{4} \mathrm{Cl}$ and found that the amplitude of the third response was severely impaired (Fig. $3 H, I$ ). Acute inhibition of dynamin activity by the application of either Dynole 34-2 or Dynasore, two structurally different dynamin inhibitors, also prevented the recovery over $1000 \mathrm{~ms}$ (Fig. 3H,I), and the role of dynamin was further corroborated by double-knockdown of endogenous dynamin 1 and 3 (Fig. $3 J-L$ ). However, inhibition of actin polymerization with latrunculin $\mathrm{B}$ did not affect the fast recovery (Fig. 3M). These data suggest that fast recovery of the RRP requires dynamin-dependent, but not actin-dependent, recycling of vesicles.

\section{Direct reuse of recycled vesicles increases during MVR}

Figure 3 showed that the fast recovery of the RRP during MVR was dependent on direct recruitment of retrieved vesicles to the RRP. Since synaptic activity can regulate the kinetics of vesicle recycling and replenishment (Wang and Kaczmarek, 1998; Sun et al., 2002; Hua et al., 2013; Delvendahl et al., 2016), the extent of direct reuse could in principle be scalable with different exocytotic mode. To test this possibility, we monitored single APevoked responses at different [Ca] in the presence of $\mathrm{NH}_{4} \mathrm{Cl}$. As described earlier, in $\mathrm{NH}_{4} \mathrm{Cl}$, recycled vesicles would remain empty and would be unable to produce iGlu response when reused, whereas already-loaded vesicles would be unaffected (Fig. 4A). Thus, the response amplitude would decrease at each trial because of the increasing proportion of glutamate-free 


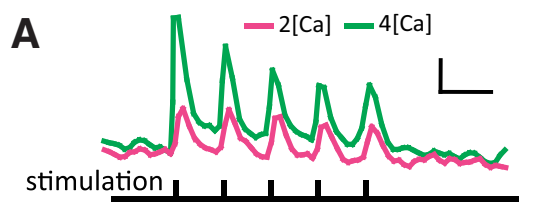

B
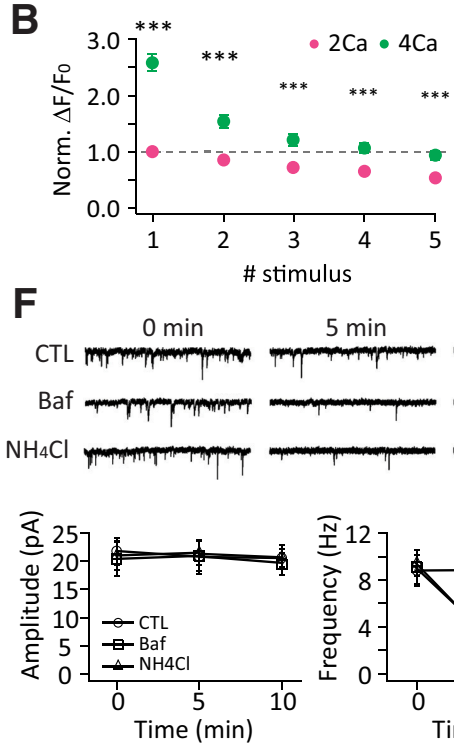

$5 \mathrm{~min}$
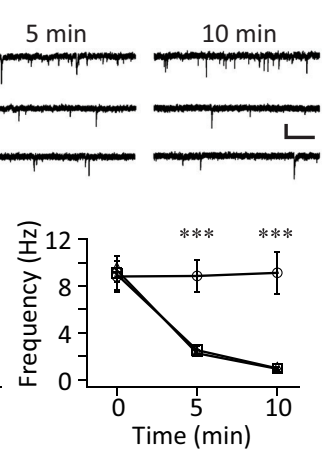

$\mathrm{H}$

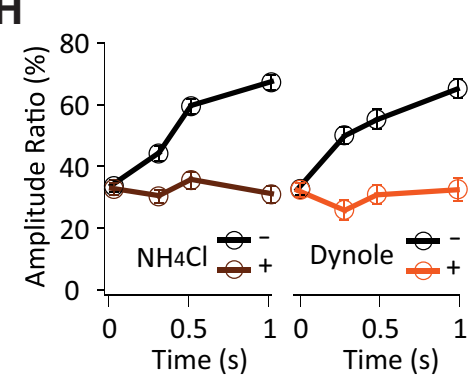

J

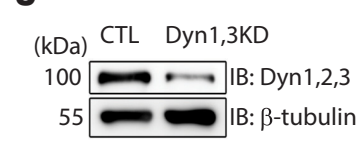

K
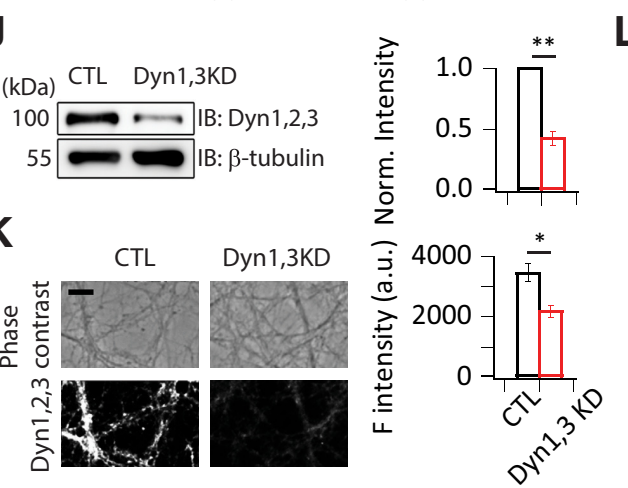

C

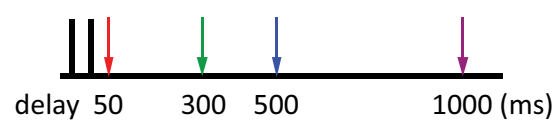

E

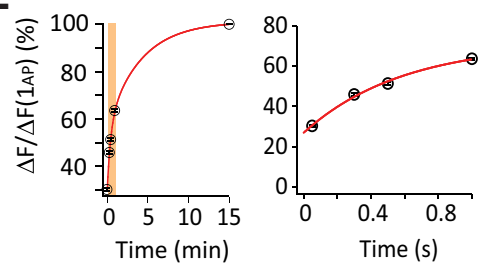

G
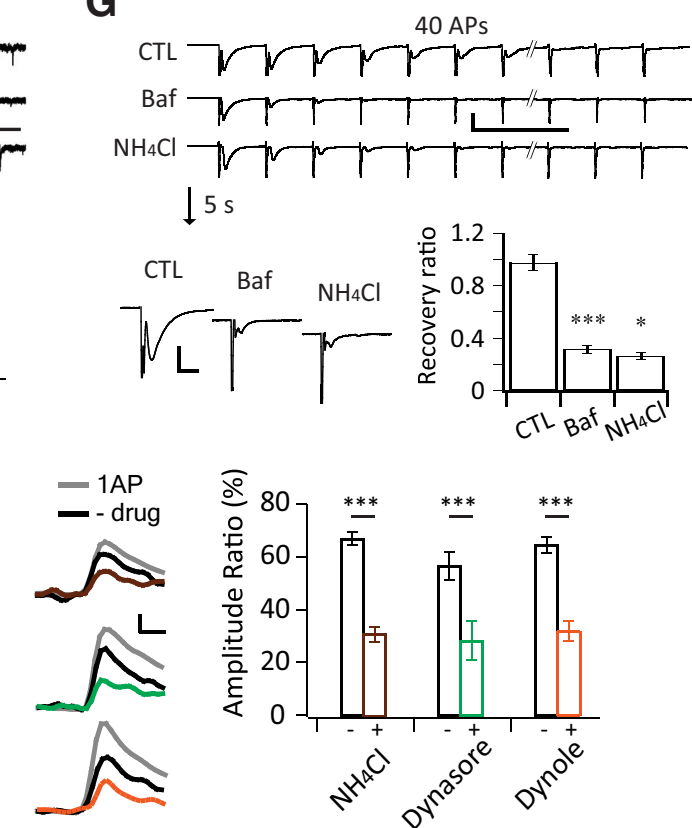

L

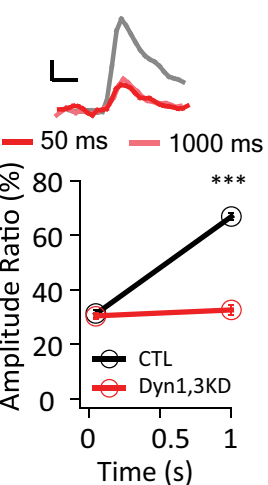

M

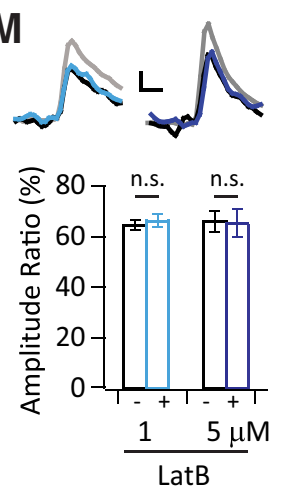

Figure 3. Dynamin-dependent fast recovery of RRP. A, Representative traces during five stimuli at $10 \mathrm{~Hz}$ at 2[Ca] (pink) and 4[Ca] (green). Each trace is an average of 10 trials. Fast decaying iGluSnFRS72A was used for precise measurement. Calibration: $50 \% \Delta \mathrm{F} / \mathrm{F}_{0}, 100 \mathrm{~ms}$. B, Pooled response amplitude at each stimulus normalized by the average of the first responses at 2[Ca]. $n=75$ boutons from 6 experiments. Mean \pm SEM: $2.57 \pm 0.10,1.53 \pm 0.08,1.20 \pm 0.07,1.06 \pm 0.06,0.94 \pm 0.06$ (4[Ca]); $1.00 \pm 0.00,0.85 \pm 0.03,0.71 \pm 0.03,0.63 \pm 0.02$, $0.52 \pm 0.02(2[C a]) . p=0.000$ for all comparisons. C, Experimental scheme to examine kinetics of recovery of the RRP. Two prestimuli at $20 \mathrm{~Hz}$ were followed by a monitoring stimulus after various delay times. $\boldsymbol{D}$, Representative evoked traces with different delays after two prestimuli. $\boldsymbol{E}$, Left, Response recovery after prestimuli as a function of delay time. Red line indicates a double exponential fit of the data. $\tau_{\text {fast }}=430.9 \mathrm{~ms}, \tau_{\text {slow }}=4.1 \mathrm{~s}$. Right, The initial $1 \mathrm{~s}$ at high resolution. $n=270$ boutons from 19 experiments. Mean \pm SEM: $30.3 \pm 0.6$ (50 ms), $45.9 \pm 0.7$ $(300 \mathrm{~ms}), 51.3 \pm 0.8(500 \mathrm{~ms}), 63.6 \pm 0.6(1000 \mathrm{~ms}) . \boldsymbol{F}$, Top, Representative traces of mEPSC at 0, 5, and 10 min in control (no treatment; (TL), $100 \mathrm{~nm}$ bafilomycin A (Baf), or $20 \mathrm{~mm} \mathrm{NH}{ }_{4} \mathrm{Cl}$. Calibration: $20 \mathrm{pA}, 0.5$ s. Bottom, Pooled amplitude (left) and frequency (right) of mEPSC in different conditions. Amplitude (mean \pm SEM, $n$ ): (TL (0 min, $21.8 \pm 2.40,6 ; 5$ min, $20.9 \pm 3.04$, 6; $10 \mathrm{~min}, 20.6 \pm 1.60,5)$, Baf (0 min, $20.4 \pm 3.00,5 ; 5 \mathrm{~min}, 21.0 \pm 2.72,11 ; 10 \mathrm{~min}, 19.5 \pm 2.00,5), \mathrm{NH}_{4} \mathrm{Cl}(0 \mathrm{~min}, 21.0 \pm 2.63,6 ; 5 \mathrm{~min}, 21.5 \pm 2.16,11 ; 10 \mathrm{~min}, 20.8 \pm 2.20,5) . p(0,5$, $10 \mathrm{~min})=0.934,0.981,0.889$. Frequency (mean $\pm \mathrm{SEM})$ : $(T L(0 \mathrm{~min}, 8.82 \pm 1.30 ; 5 \mathrm{~min}, 8.86 \pm 1.37 ; 10 \mathrm{~min}, 9.13 \pm 1.80)$, Baf $(0 \mathrm{~min}, 9.10 \pm 1.43 ; 5 \mathrm{~min}, 2.52 \pm 0.33 ; 10 \mathrm{~min}$, $0.95 \pm 0.10), \mathrm{NH}_{4} \mathrm{Cl}(0 \mathrm{~min}, 9.47 \pm 1.09 ; 5 \mathrm{~min}, 2.25 \pm 0.26 ; 10 \mathrm{~min}, 0.91 \pm 0.14) . p(0,5,10 \mathrm{~min})=0.932,0.000,0.000$ (one-way ANOVA test). $G$, Representative traces of EPSC during 40 $\mathrm{AP}$ at $20 \mathrm{~Hz}$ (top) and $1 \mathrm{AP}$ in $5 \mathrm{~s}$ recovery (bottom left) in different conditions. Bottom right, Pooled recovered response by $1 \mathrm{AP}$ normalized by the first response during $40 \mathrm{AP}$ in different conditions. Mean \pm SEM $(n): 0.98 \pm 0.06$ (CTL, 5), $0.32 \pm 0.03$ (Baf, 4), $0.26 \pm 0.03\left(\mathrm{NH}_{4} \mathrm{Cl}, 4\right) . p\left(\mathrm{CTL}, \mathrm{Baf}, \mathrm{NH}_{4} \mathrm{Cl}\right)=(0.303,0.039,0.045)$, paired Student $t$ test. Calibration: $1 \mathrm{nA}, 100 \mathrm{~ms}(40$ APs), $10 \mathrm{~ms}$ (1 AP). $\boldsymbol{H}$, Response recovery after prestimuli as a function of delay time in the presence of $\mathrm{NH}_{4} \mathrm{Cl}$ (left, brown) or Dynole (right, orange). $n(50,300,500,1000 \mathrm{~ms})=(147,94,101$, 105) boutons from $(12,10,11,9)$ experiments in $\mathrm{NH}_{4} \mathrm{Cl} ;(96,60,66,61)$ boutons from $(11,8,8,7)$ experiments in Dynole. $p(50,300,500,1000 \mathrm{~ms})=(0.453,0,0,0)$ in $\mathrm{NH}_{4} \mathrm{Cl} ;(0.869,0,0,0)$ 
A

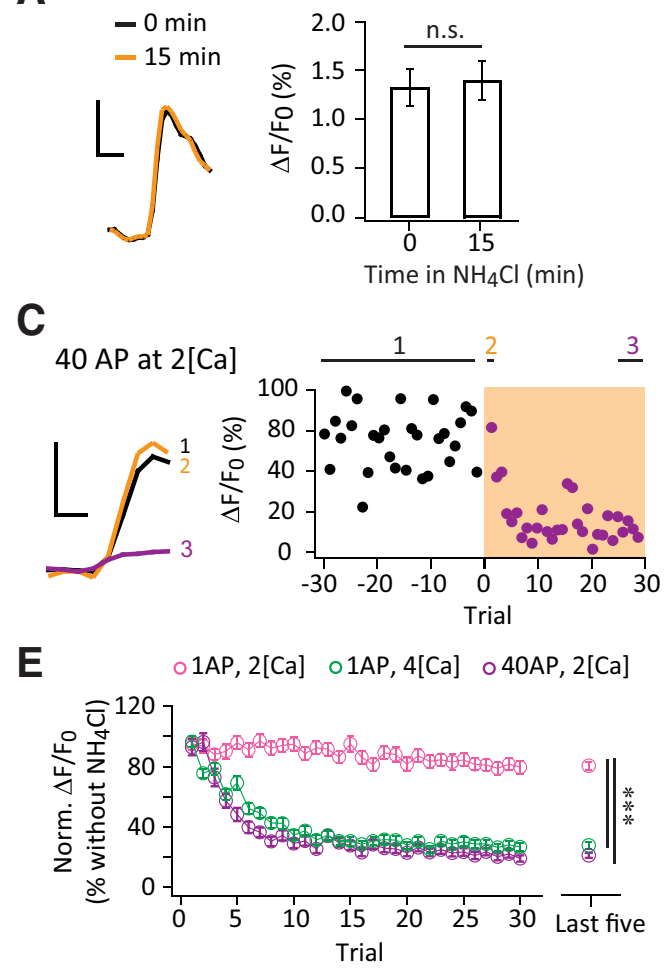

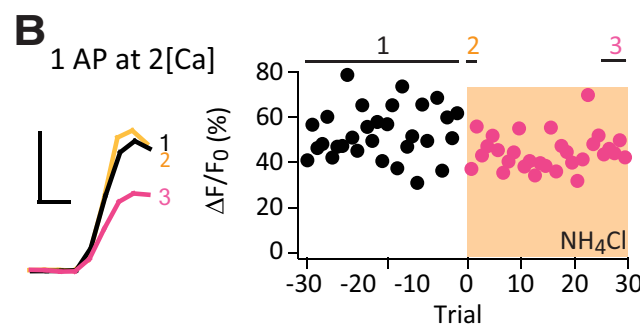

D

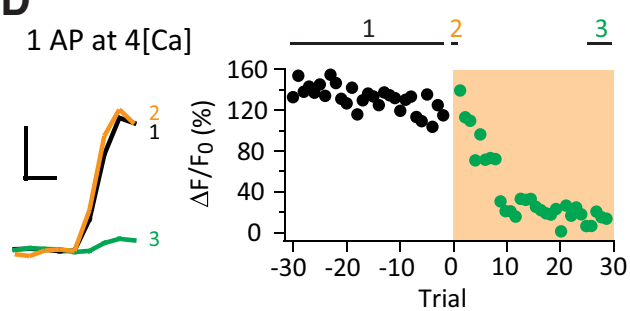

$F$

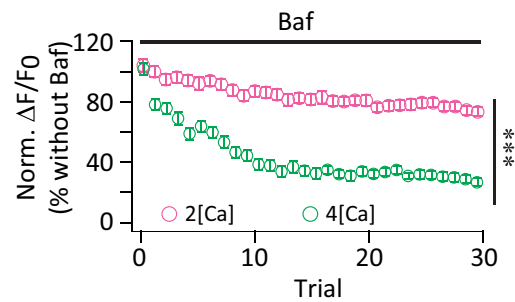

Figure 4. The extent of direct reuse is scalable with different exocytotic activity. $\mathbf{A}$, Left, Representative responses to $1 \mathrm{AP}$ for 0 and $15 \mathrm{~min}$ in $\mathrm{NH}_{4} \mathrm{Cl}$. Calibration: $50 \% \Delta \mathrm{F} / \mathrm{F}_{0}, 50 \mathrm{~ms}$. Right, Pooled graph of response amplitude in $\mathrm{NH}_{4} \mathrm{Cl}$ in 0 and $15 \mathrm{~min} . n=8$ experiments. Mean \pm SEM: $1.32 \pm 0.19$ ( $0 \mathrm{~min}$ ), $1.39 \pm 0.20$ (15 min). $p=0.79490$ (paired Student's $t$ test). $\boldsymbol{B}$ - $\boldsymbol{D}$, Changes in the amount of glutamate released in the presence of $20 \mathrm{mM} \mathrm{NH}_{4} \mathrm{Cl}$ in response to $1 \mathrm{AP}$ at 2[Ca] (B), $1 \mathrm{AP}$ at 4[Ca] (C), and 40AP at 2[Ca] (D), repeated for 30 trials. Left, Representative traces from individual presynaptic terminals in each group. The average response without $\mathrm{NH}_{4} \mathrm{Cl}(1)$, the first successful response (2), and the average of the last five responses (3) in the presence of $\mathrm{NH}_{4} \mathrm{Cl}$. Calibration: $50 \% \Delta \mathrm{F} / \mathrm{F}_{0}, 20 \mathrm{~ms}$. Right, Response amplitude as a function of time. Yellow shade represents the presence of $\mathrm{NH}_{4} \mathrm{Cl}$ in the bath solution. The amplitude of the response to the first AP during the sets of $40 \mathrm{AP}$ trials in $\mathrm{NH}_{4} \mathrm{Cl}$ was measured. $\boldsymbol{E}$, Pooled response amplitude in each group in the presence of $\mathrm{NH}_{4} \mathrm{Cl}$. $n($ bouton, experiment) $=$ $(165,7)$ for $2[\mathrm{Ca}]$, 40AP; $(206,7)$ for 4[Ca], $1 \mathrm{AP} ;(239,10)$ for 2[Ca], 1 AP. Data were normalized to the responses without $\mathrm{NH}_{4} \mathrm{Cl}$. Last five (mean \pm SEM): $0.210 \pm 0.016(2[\mathrm{Ca}]$, $40 \mathrm{AP})$, $0.275 \pm 0.021$ (4[Ca], $1 \mathrm{AP}), 0.805 \pm 0.029$ (2[Ca], $1 \mathrm{AP}) . p(1 \mathrm{AP}$ at 2[Ca], 40AP at 2[Ca]) $=0.000, p(1 \mathrm{AP}$ at 2[Ca], $1 \mathrm{AP}$ at 4[Ca]) $=0.000, p(40 \mathrm{AP}$ at 2[Ca], $1 \mathrm{AP}$ at $4[\mathrm{Ca}])=0.630$ (Student's $t$ test). $\boldsymbol{F}$, Pooled response amplitude by $1 \mathrm{AP}$ at $2[\mathrm{Ca}]$ and $4[\mathrm{Ca}]$ in Baf. $n$ (bouton, experiment) $=(192,10)$ for 2[Ca], $(134,10)$ for 4[Ca]. $p=0.000$ (two-way repeated-measures ANOVA). $* * * 0.001$

vesicles in the RRP. While iGlu responses declined slowly over the trials at $2[\mathrm{Ca}]$, they decayed dramatically at $4[\mathrm{Ca}](\mathrm{Fig} .4 B, D$, E). When $40 \mathrm{APs}$ were used at $2[\mathrm{Ca}]$ to deplete the RRP at each trial, the iGlu response to the first AP also declined fast (Fig. 4C).

in Dynole. Data are mean $\pm 95 \%$ Cl. $I$, Left, Representative response to 1 AP (an average of 20 trials, gray), 1 AP with $1000 \mathrm{~ms}$ delay after prestimuli without (black) or with drugs (Dynasore, green). Right, Pooled response recovery $1000 \mathrm{~ms}$ after prestimuli in the presence of different drugs. $n$ (Dynasore $)=64$ boutons from 6 experiments. $p$ (Dynasore) $=0.000 . J$, Left, Western blot from control (CTL) and siRNA of dynamin1,3-transfected neurons (Dyn1,3KD) detected by antibody against dynamin1,2,3 (Dyn1,2,3). Right, Pooled band intensity normalized by CTL. $n=5$ for each group. $p=0.002$ (paired Student's $t$ test). $\boldsymbol{K}$, Left, Representative images from (TL and Dyn1,3KD immunostained by antibody against Dyn1,2,3. Scale bar, $10 \mu \mathrm{m}$. Right, Pooled fluorescent intensity in neurites. $n=8$ (CTL), 5 (Dyn1,3KD). $p=0.015$ (Student's $t$ test). L, Top, Representative response to 1 AP (gray), 1 AP with 50 (red) and 1000 (salmon) ms delay after prestimuli. Bottom, Pooled response recovery in $C T L$ and Dyn1,3KD. $n$ (bouton, experiment, delay) $=(78,6,50)$ and $(78,6,1000)$ for $C T L$, $(57,4,50)$ and $(95,8,1000)$ for Dyn1,3KD. $p(C \mathrm{CL}$, Dyn1,3KD) $=0.782$ for $50 \mathrm{~ms}, 0.00000$ for $1000 \mathrm{~ms}$. $p(50,1000)$ for Dyn1,3KD $=0.375$. $M$, Top, Representative response to 1 AP (gray), 1 AP with $1000 \mathrm{~ms}$ delay after prestimuli without (black) or with latrunculin B (LatB). Light blue represents $1 \mu \mathrm{m}$. Dark blue represents $5 \mu \mathrm{m}$. Bottom, Pooled response recovery $1000 \mathrm{~ms}$ after prestimuli in 1 and $5 \mu \mathrm{m}$ LatB. $n$ (bouton, experiment) $=(152,11)$ in $1 \mu \mathrm{m}$; $(51,5)$ in $5 \mu \mathrm{M} . p(1 \mu \mathrm{M})=0.171, p(5 \mu \mathrm{m})=0.763$. Data are mean $\pm 95 \%$ Cl. ${ }^{*} p<0.05$; ${ }^{* *} p<0.01$; ${ }^{* * *} p<0.001$; paired Student's $t$ test. Calibration: $\boldsymbol{D}, I, L, M, 50 \% \Delta \mathrm{F} / \mathrm{F}_{0}$, $50 \mathrm{~ms}$.
We repeated the experiment in the presence of Baf and found comparable results. (Fig. 4F). These data suggest that the extent of recycled vesicle recruitment to the RRP depends on the exocytotic activity.

The kinetics of glutamate depletion in Figure $4 E$ is determined by two factors: the number of vesicles released per AP and the proportion of empty vesicles directly recruited to the RRP (Fig. 5). In the presence of $\mathrm{NH}_{4} \mathrm{Cl}$, the retrieved glutamatefree vesicles would be distributed either to the RRP for immediate release or to the RP for later replenishment of the RRP. Thus, the more vesicles released, the larger the fraction of the RRP refilled by empty vesicles, resulting in faster depletion. Moreover, the higher the proportion of vesicles reused, the faster would glutamate depletion occur even if the same number of vesicles were released per trial. Obviously, the number of vesicles released per AP is larger at $4[\mathrm{Ca}]$ than $2[\mathrm{Ca}]$, as shown in Figures 1, 2, and 4, yet whether or not the proportion of vesicles directly recruited to the RRP changes cannot be conclusively determined.

To investigate the contribution of direct reuse, we constructed a simple quantitative model to estimate parameters that best fit the empirical data during different release modes (Fig. 6A; for more details, see Materials and Methods, Discussion). Briefly, the model comprises four parameters: $\mathrm{N}_{\mathrm{RP}}, \mathrm{N}_{\mathrm{RRP}}, \mathrm{Pv}$, and the proportion of the retrieved vesicles directly reused in the RRP $(\mathrm{Pe})$. With this set of four parameters, we simulated the amount of glutamate released in response to 30 trials of $1 \mathrm{AP}$ as 
A The number of released vesicles per AP

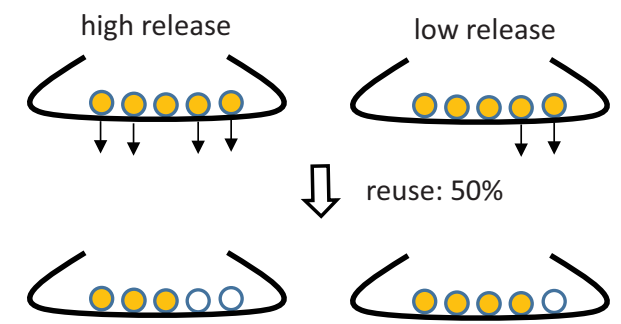

B The proportion of retrieved vesicles diretly reused to the RRP

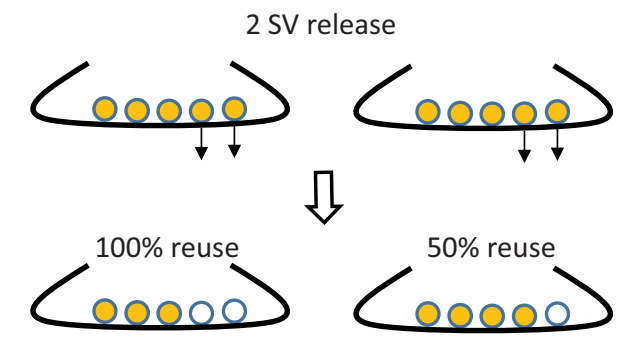

Figure 5. Schematics of two determinants of glutamate depletion in Figure 4. Schematics that illustrate how the number of released vesicles per AP $(A)$ and the proportion of retrieved vesicles directly reused to the RRP $(\boldsymbol{B})$ determine the shape of glutamate decay in Figure 4. Yellow circles represent glutamate-filled vesicles. Open circles represent empty vesicles in $\mathrm{NH}_{4} \mathrm{Cl}$ (for details, see Results).

performed in the experiment and calculated the set of best fit data (see Materials and Methods). Then, we changed one parameter while the other three were fixed, and evaluated the effect on the goodness of fit of the simulated data to the empirical data (Fig. $6 B)$. At $2[\mathrm{Ca}]$, a $\mathrm{Pe}<0.2$ ( 0.1 for the best fit) made the simulated data a fairly good fit to the empirical data at $2[\mathrm{Ca}]$ (Fig. 6B,C). In this condition, $\mathrm{Pv}>0.5$ seemed to affect the fit of the simulated data, yet this value is too high to be realistic at $2[\mathrm{Ca}]$ and thus was disregarded. On the other hand, at $4[\mathrm{Ca}]$, Pe for the best fit was 0.7 , and the simulated data no longer represented the empirical data when a small change was made in Pe. $\mathrm{N}_{\mathrm{RRP}}$ and $\mathrm{N}_{\mathrm{RP}}$ did not affect the simulation results in either condition. To estimate the relevant range of $\mathrm{Pe}$, we sorted the parameter sets by their $\chi^{2}$ values, collected the top $1 \%$ of the total parameter sets (101 of 10,080), and plotted the distribution histogram (Fig. 6D). Interestingly, the distributions of $\mathrm{Pe}$ at $2[\mathrm{Ca}]$ and $4[\mathrm{Ca}]$ were clearly separated, with the mean at $4[\mathrm{Ca}](0.681)$, almost 10 times higher than that at $2[\mathrm{Ca}](0.068)$. The distributions of the other three parameters were not significantly different at the different [Ca]s. These findings suggest that the proportion of recycled vesicles directly reused in the RRP is dynamically regulated, increasing up to 10 -fold with the number of vesicles released.

\section{Discussion}

Our experiments using iGluSnFRpre and simulations have provided evidence that hippocampal synapses are capable of releasing multiple vesicles in response to an AP, and that when MVR becomes prevalent, the contribution of vesicles directly recruited to the RRP dramatically increases. These results reveal a form of presynaptic homeostatic plasticity that allows reliable synaptic transmission during different types of synaptic activity.

\section{MVR and docking sites}

Spontaneous and evoked responses measured using iGluSnFRpre showed that hippocampal synapses release glutamate in a quantal manner, and are capable of releasing multiple vesicles simultaneously depending on the extracellular calcium level. Although the occurrence of MVR seems incompatible with some electrophysiological studies supporting the single-vesicle hypothesis, more recent spatially precise measurements from individual synapses using the calcium indicator, GCaMP, or another glutamate sensor, eEOS, are in agreement with our data (Oertner et al., 2002; Sakamoto et al., 2018).

The observation of MVR indicates that there are multiple docking sites in the active zone. A docking site is a location where a vesicle can be docked and actual vesicle release takes place, and their number is thus a key determinant of synaptic strength. Indeed, differences in docking site numbers between synapses appear to be the major factor underlying intersynaptic variability (Auger and Marty, 2000). We found that, at $4[\mathrm{Ca}]$, the maximal evoked response to $1 \mathrm{AP}$ was $\sim 0.6$ of the RRP (Fig. $2 I$ ). Since an increase in [Ca] to $8 \mathrm{~mm}$ did not induce further release (Fig. $2 L$ ), we were able to estimate the relationship between docking site number and size of the RRP using the data obtained at 4 [Ca]. If all the docking sites were occupied, the size of the RRP would be up to twofold higher than the docking site number. Alternatively, if the size of the RRP and the number of release sites is nearly equivalent, as suggested by a recent study (Sakamoto et al., 2018), our data suggest that $\sim 60 \%$ of the docking sites are occupied by vesicles. This result is close to the value $(0.45)$ obtained for the synapses between cerebellar parallel fibers and molecular layer interneurons (Auger and Marty, 2000).

\section{The proportion of direct vesicle reuse after exocytosis}

Direct reuse of the limited number of vesicles in the RRP can expand the functional capabilities of small central synapses during robust repetitive AP firing. Especially during MVR when the RRP is depleted fast, rapid replenishment of the RRP could be critical for maintaining synaptic transmission. However, it has never been reliably observed whether direct reuse occurs in response to a single AP and, if so, how much it contributes to a subsequent release. This is mainly because previous optical reporters require trains of APs to produce detectible signals and during that time the RRP is refilled not only by reuse but also by $\mathrm{RP}$-originated vesicles. In contrast, iGluSnFR, which responds reliably to a single AP, allowed us to observe two previously unrevealed phenomena during synaptic vesicle recycling. First, we were able to distinguish two kinetically distinct components of vesicle recycling and found that fast recovery with a time constant of $430 \mathrm{~ms}$ occurs via a dynamin-dependent mechanism. Second, we could calculate the ratio of reused and RP-originated vesicles in the RRP after each single AP. Because there was a sufficient interval between each trial, we were able to create a quantitative model that reflected the proportion of glutamate-filled vesicles that contribute to a subsequent release, without taking account of the kinetic parameters of the individual processes that occur during vesicle recycling. By manipulating the number of vesicles released at each trial by varying [Ca], we found that $\sim 70 \%$ of freshly released vesicles were directly recruited to the $\mathrm{RRP}$ at $4[\mathrm{Ca}]$; that is a larger proportion than reported previously, but similar to the fraction of kiss-and-run events during hypertonic application (80\%) (Stevens and Williams, 2000). In contrast, the proportion of direct reuse dropped to $7 \%$ at $2[\mathrm{Ca}]$, suggesting that the contribution of direct reuse to RRP replenishment increases significantly with the number of vesicles released.

A previous study suggested that, because of the relatively slow glutamate refilling rate $\left(\sim 15 \mathrm{~s}\right.$ at room temperature, $\sim 7 \mathrm{~s}$ at $35^{\circ}$ C), the contribution of rapidly reused vesicles to synaptic 
A

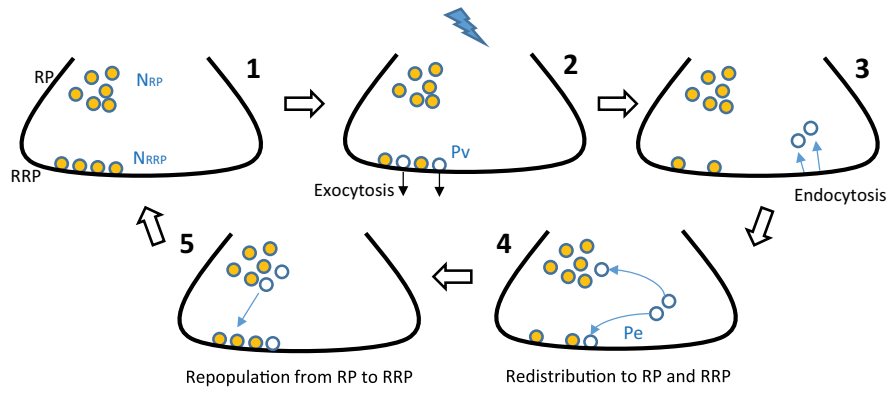

B

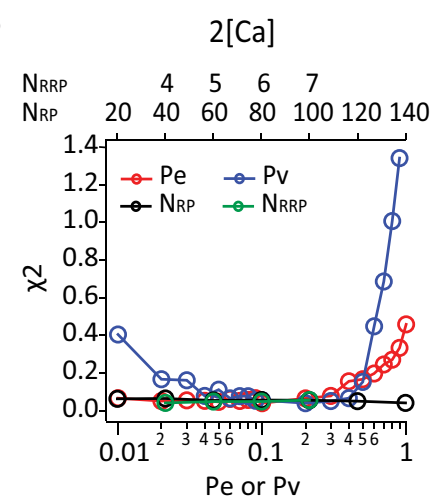

$4[\mathrm{Ca}]$

$\begin{array}{llll}4 & 5 & 6 & 7\end{array}$

$20 \quad 40 \quad 60 \quad 80100120140$

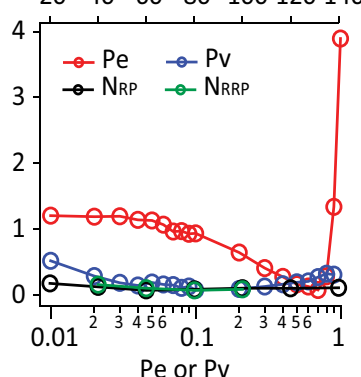

D

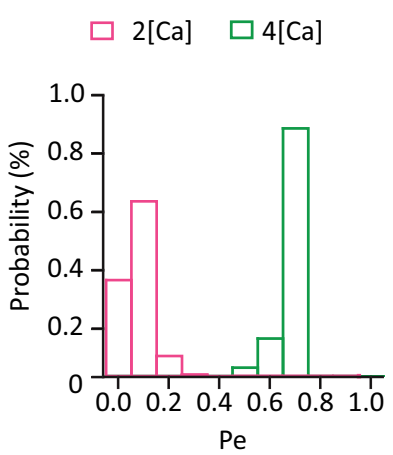

C

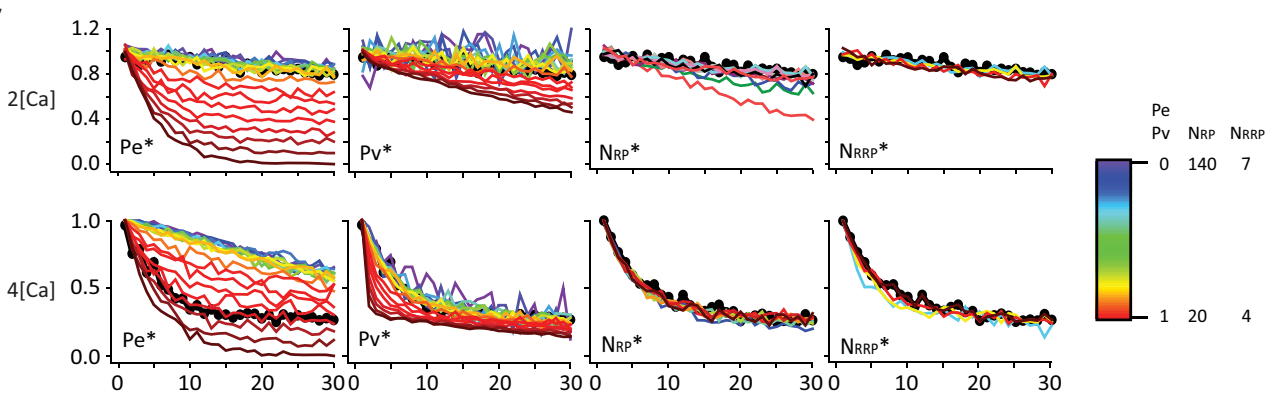

Figure 6. Direct reuse of recycled vesicles increases during MVR. A, Schematics of the computational model for the experiment in Figure 4. Yellow circles represent vesicles filled with glutamate. Open circles represent vesicles without glutamate (for full description, see Materials and Methods). $\boldsymbol{B}$, Minimal differences between empirical and simulated data $\left(\chi^{2}\right)$ for each parameter required for modeling the experiments at 2[Ca] (left) and 4[Ca] (right). $C$, Simulated data with one parameter (marked with *) changing while the other three fixed with the values for the best fit at 2[Ca] $(\boldsymbol{A})$ and $4[\mathrm{Ca}](\boldsymbol{B})$. Color code indicates the parameter values. $\boldsymbol{D}$, The distributions of Pe in the top $1 \%$ of the total parameter sets sorted by the $\chi^{2}$ at $2[\mathrm{Ca}]$ and $4[\mathrm{Ca}]$.

transmission would be limited (Hori and Takahashi, 2012). However, we believe that direct comparisons between the giant synapses of the calyces and the small hippocampal synapses should be made with caution for the following reasons: (1) Each calyx of Held synapse contains $>300$ active zones and $>300,000$ vesicles, with an RRP estimated at $>2000$ vesicles (Qiu et al., 2015), while a single hippocampal synapse contains $\sim 50-100$ vesicles with an RRP of $\sim 5$ vesicles (i.e., a $>3000$-fold difference). (2) The cytosolic glutamate concentration is the major determinant of vesicular refilling rate. Although UV photolysis of caged glutamate increases cytosolic glutamate rapidly, the effectiveness of photolysis and the cytosolic glutamate concentration after photolysis are unknown. Considering the size and number of synaptic vesicles that would compete to refill glutamate in the calyx synapses, their estimates could be at the lower limit of the glutamate refilling rate. (3) We have shown that, after the release of a substantial portion of the RRP by consecutive $20 \mathrm{~Hz}$ APs at 4 [Ca], iGluSnFR responses recovered by up to $\sim 63.8 \%$ of the initial response with a $\tau_{\text {fast }}$ of $430 \mathrm{~ms}$, followed by a slow component with $\tau=4.11 \mathrm{~s}$ at $35^{\circ} \mathrm{C}$. The experimental scheme and time resolution used by Hori and Takahashi (2012), however, were not able to reveal the fast component of recovery at the level of seconds. Indeed, their estimate of the refilling rate of $\tau=7 \mathrm{~s}$ at $35^{\circ} \mathrm{C}$ is not very different from our estimate for the slow component of recovery $(4.11 \mathrm{~s})$. (4) A recent study found that $\mathrm{Na}^{+}$ accumulation in the calyx of Held presynaptic terminals during high-frequency signaling activates $\mathrm{Na}^{+} / \mathrm{H}^{+}$exchangers, which significantly accelerate glutamate refilling (Li et al., 2020). We expect that the impact of $\mathrm{Na}^{+}$on vesicle refilling at the small size of hippocampal synapses would be higher and thus further facilitate refilling. (5) Finally, unlike the calyx synapses, the small CNS synapses could have evolved a rapid reuse mechanism to counteract the limited number of vesicles available to sustain synaptic transmission (Harata et al., 2001).

\section{The mechanism underlying rapid direct reuse}

We found that the early recovery response had a very rapid time constant $(430 \mathrm{~ms})$, much faster than previously reported (Pyle et al., 2000; Sara et al., 2002). A previous study using the calyx of Held synapse showed that, after depletion of the RRP, slowly releasing vesicles recover rapidly, whereas rapidly releasing vesicles recover slowly (Sakaba and Neher, 2001). The authors also found that the recovery of rapidly releasing vesicles was facilitated by $\mathrm{Ca}^{2+}$, which is consistent with our results. Interestingly, their estimates of the rates of the fast and slow components of RRP refilling are similar to 
ours (196 ms vs $430 \mathrm{~ms}$ for the fast component; $4.5 \mathrm{~s}$ vs $4.11 \mathrm{~s}$ for the slow component). Despite the order of magnitude differences in the size of RRP, type of synapses, and experimental procedure, these results point to the possibility of a common mechanism governing rapid vesicle reuse involving vesicular release probability and $\mathrm{Ca}^{2+}$. Although we are not sure whether the rapidly reused vesicles that are increased in proportion in our MVR observations are the same as the rapidly releasing vesicles whose refilling was facilitated by $\mathrm{Ca}^{2+}$ in their study, it would be interesting to study whether vesicles with different release probabilities contribute differently to rapid vesicle reuse.

How is this fast recovery achieved? We showed that it is mediated by dynamin-dependent endocytosis of previously released vesicles, excluding the contribution of RP-originated vesicles. Of the various endocytic mechanisms suggested, the one that can best explain the short timescale and drastic decrease in response size in our results is the kissand-run mechanism (Stevens and Williams, 2000; He et al., 2006). Although the recently discovered ultrafast endocytosis occurs on a timescale of $\sim 100 \mathrm{~ms}$, it requires actin polymerization and takes an additional 5-10 $\mathrm{s}$ to produce releasable vesicles (Watanabe et al., 2014); thus, it does not account for our results. Recent studies suggest that release site clearance may facilitate mobilization of vesicles to the RRP (Hosoi et al., 2009; X. S. Wu et al., 2009). Release site clearance is a process that rapidly clears the exocytosed material from the fusion site toward the periactive zone where endocytic retrieval occurs to enable new rounds of vesicle docking and release (Neher, 2010; L. G. Wu et al., 2014). Thus, rapid reuse may depend on the clearance of released components to the periactive zone followed by dynamin-dependent endocytosis.

The underlying mechanism still needs to be clarified, yet direct reuse surely plays a role depending on the mode of exocytosis. In the calyx of Held, rapidly recycled vesicles are recruited not to the RRP, but to the RP (X. S. Wu and Wu, 2009). Therefore, direct transfer to the RRP may be a presynaptic mechanism designed to sustain synaptic transmission in synapses with small vesicle pools. In conclusion, our experiments using iGluSnFRpre have resolved the contribution of direct recruitment of retrieved vesicles to the RRP in response to a single AP and showed that the extent of direct recruitment is dynamically modulated depending on the number of vesicles released. Since the usage of synaptic vesicles is determined by various presynaptic activities and direct reuse of recycled vesicles takes place within a few hundred milliseconds, varying the contribution of direct reuse could be short-term homeostatic plasticity that ensures the presence of releasable vesicles in the RRP for reliable synaptic transmission over a range of exocytotic modes.

\section{References}

Auger C, Marty A (2000) Quantal currents at single-site central synapses. J Physiol 526:3-11.

Biro AA (2005) Quantal size is independent of the release probability at hippocampal excitatory synapses. J Neurosci 25:223-232.

Bolshakov VY, Siegelbaum SA (1995) Regulation of hippocampal transmitter release. Science 269:1730-1734.

Chamberland S, Evstratova A, Toth K (2014) Interplay between synchronization of multivesicular release and recruitment of additional release sites support short-term facilitation at hippocampal mossy fiber to CA3 pyramidal cells synapses. J Neurosci 34:11032-11047.
Chen G, Harata NC, Tsien RW (2004) Paired-pulse depression of unitary quantal amplitude at single hippocampal synapses. Proc Natl Acad Sci USA 101:1063-1068.

Conti R, Lisman J (2003) The high variance of AMPA receptor- and NMDA receptor-mediated responses at single hippocampal synapses: evidence for multiquantal release. Proc Natl Acad Sci USA 100:4885-4890.

Delvendahl I, Vyleta NP, Hallermann S, Delvendahl I, Vyleta NP, Gersdorff H, Von Hallermann S (2016) Independent endocytosis at central synapses endocytosis at central synapses. Neuron 90:492-498.

Ertunc M, Sara Y, Chung C, Atasoy D, Virmani T, Kavalali ET (2007) Fast synaptic vesicle reuse slows the rate of synaptic depression in the CA1 region of hippocampus. J Neurosci 27:341-354.

Fernández-Alfonso T, Ryan TA (2004) The kinetics of synaptic vesicle pool depletion at CNS synaptic terminals. Neuron 41:943-953.

Granseth B, Lagnado L (2008) The role of endocytosis in regulating the strength of hippocampal synapses. J Physiol 586:5969-5982.

Granseth B, Odermatt B, Royle SJ, Lagnado L (2007) Clathrin-mediated endocytosis: the physiological mechanism of vesicle retrieval at hippocampal synapses. J Physiol 585:681-686.

Hanse E, Gustafsson B (2001) Vesicle release probability and pre-primed pool at glutamatergic synapses in area CA1 of the rat neonatal hippocampus. J Physiol 531:481-493.

Harata N, Pyle JL, Aravanis AM, Mozhayeva M, Kavalali ET, Tsien RW (2001) Limited numbers of recycling vesicles in small CNS nerve terminals: implications for neural signaling and vesicular cycling. Trends Neurosci 24:637-643.

Harata NC, Aravanis AM, Tsien RW (2006) Kiss-and-run and full-collapse fusion as modes of exo-endocytosis in neurosecretion. J Neurochem 97:1546-1570

He L, Wu XS, Mohan R, Wu LG (2006) Two modes of fusion pore opening revealed by cell-attached recordings at a synapse. Nature 444:102-105.

Hori T, Takahashi T (2012) Kinetics of synaptic vesicle refilling with neurotransmitter glutamate. Neuron 76:511-517.

Hosoi N, Holt M, Sakaba T (2009) Calcium dependence of exo- and endocytotic coupling at a glutamatergic synapse. Neuron 63:216-229.

Hua Y, Woehler A, Kahms M, Haucke V, Neher E, Klingauf J (2013) Blocking endocytosis enhances short-term synaptic depression under conditions of normal availability of vesicles. Neuron 80:343-349.

Li D, Zhu Y, Huang H (2020) Spike activity regulates vesicle filling at a glutamatergic synapse. J Neurosci 40:4972-4980.

Marvin JS, Scholl B, Wilson DE, Podgorski K, Kazemipour A, Müller JA, Schoch S, Quiroz FJ, Rebola N, Bao H, Little JP, Tkachuk AN, Cai E, Hantman AW, Wang SS, DePiero VJ, Borghuis BG, Chapman ER, Dietrich D, DiGregorio DA, et al. (2018) Stability, affinity, and chromatic variants of the glutamate sensor iGluSnFR. Nat Methods 15:936-939.

Marvin JS, Borghuis BG, Tian L, Cichon J, Harnett MT, Akerboom J, Gordus A, Renninger SL, Chen TW, Bargmann CI, Orger MB, Schreiter ER, Demb JB, Gan WB, Hires SA, Looger LL (2013) An optimized fluorescent probe for visualizing glutamate neurotransmission. Nat Methods 10:162170.

Neher E (2010) What is rate-limiting during sustained synaptic activity: vesicle supply or the availability of release sites. Front Synaptic Neurosci 2:1-6.

Oertner TG, Sabatini BL, Nimchinsky EA, Svoboda K (2002) Facilitation at single synapses probed with optical quantal analysis. Nat Neurosci 5:657664.

Pyle JL, Kavalali ET, Piedras-Rentería ES, Tsien RW (2000) Rapid reuse of readily releasable pool vesicles at hippocampal synapses. Neuron 28:221-231.

Qiu X, Zhu Q, Sun J (2015) Quantitative analysis of vesicle recycling at the calyx of Held synapse. Proc Natl Acad Sci USA 112:4779-4784.

Sakaba T, Neher E (2001) Calmodulin mediates rapid recruitment of fastreleasing synaptic vesicles at a calyx-type synapse. Neuron 32:1119-1131.

Sakamoto H, Ariyoshi T, Kimpara N, Sugao K, Taiko I, Takikawa K, Asanuma D, Namiki S, Hirose K (2018) Synaptic weight set by Munc13-1 supramolecular assemblies. Nat Neurosci 21:41-55.

Sara Y, Mozhayeva MG, Liu X, Kavalali ET (2002) Fast vesicle recycling supports neurotransmission during sustained stimulation at hippocampal synapses. J Neurosci 22:1608-1617.

Schikorski T, Stevens CF (1997) Quantitative ultrastructural analysis of hippocampal excitatory synapses. J Neurosci 17:5858-5867. 
Silver RA, Lubke J, Sakmann B, Feldmeyer D (2003) High-probability uniquantal transmission at excitatory synapses in barrel cortex. Science 302:1981-1985.

Stevens CF, Wang Y (1995) Facilitation and depression at single central synapses. Neuron 14:795-802.

Stevens CF, Williams JH (2000) Kiss and run exocytosis at hippocampal synapses. Proc Natl Acad Sci 97:12828-12833.

Sun JY, Wu XS, Wu LG (2002) Single and multiple vesicle fusion induce different rates of endocytosis at a central synapse. Nature 417:555-559.

Tong G, Jahr CE (1994) Multivesicular release from excitatory synapses of cultured hippocampal neurons. Neuron 12:51-59.

Wadiche JI, Jahr CE (2001) Multivesicular release at climbing fiber-Purkinje cell synapses. Neuron 32:301-313.

Wang LY, Kaczmarek LK (1998) High-frequency firing helps replenish the readily releasable pool of synaptic vesicles. Nature 394:384-388.
Watanabe S, Trimbuch T, Camacho-Pérez M, Rost BR, Brokowski B, SöhlKielczynski B, Felies A, Davis MW, Rosenmund C, Jorgensen EM (2014) Clathrin regenerates synaptic vesicles from endosomes. Nature 515:228233.

Wu LG, Hamid E, Shin W, Chiang HC (2014) Exocytosis and endocytosis: modes, functions, and coupling mechanisms. Annu Rev Physiol 76:301331.

Wu XS, Wu LG (2009) Rapid endocytosis does not recycle vesicles within the readily releasable pool. J Neurosci 29:11038-11042.

Wu XS, McNeil BD, Xu J, Fan J, Xue L, Melicoff E, Adachi R, Bai L, Wu LG (2009) $\mathrm{Ca}^{2+}$ and calmodulin initiate all forms of endocytosis during depolarization at a nerve terminal. Nat Neurosci 12:1003-1010.

Zhang Q, Li Y, Tsien RW (2009) The dynamic control of kiss-and-run and vesicular reuse probed with single nanoparticles. Science 323:1448-1453. 